\title{
Analysis of high frequency vibrations and heat impact on human hands
}

\author{
Andrius Romualdas Juknevičiuss', Mantas Venslauskas², Paulina Jolanta Žilinskaitė \\ Edvinas Litvinas ${ }^{4}$, Algimantas Bubulis ${ }^{5}$, Asta Baranauskaité ${ }^{6}$ \\ ${ }^{1,4}$ Kaunas University of Technology, Faculty of Electrical and Electronics Engineering, Kaunas, Lithuania \\ ${ }^{2,5}$ Kaunas University of Technology, Institute of Mechatronics, Kaunas, Lithuania \\ ${ }^{3,6}$ Hospital of Lithuanian University of Health Sciences Kauno Klinikos, Department of Rheumatology, \\ Kaunas, Lithuania \\ ${ }^{1}$ Corresponding author \\ E-mail: ${ }^{1}$ andrius.juknevicius@ktu.edu, ${ }^{2}$ mantas.venslauskas@ktu.edu, ${ }^{3}$ zilinskaite.pj@gmail.com, \\ 4edvinas.litvinas@ktu.edu, ${ }^{5}$ algimantas.bubulis@ktu.lt, ${ }^{6}$ astai@centras.lt
}

Received 8 November 2017; accepted 15 November 2017

DOI https://doi.org/10.21595/vp.2017.19418

Check for updates

\begin{abstract}
This article describes combined impact of ultrasound and heat therapies on patients with rheumatoid arthritis, systemic scleroderma and Raynaud's syndrome. Theoretical background of ultrasound and thermal effect was analyzed and described. Flexibility, strength and temperature of subjects' wrists and hands were investigated using thermographic camera, dynamometer, and physiological indicators. Investigation results were analyzed and demonstrated in graphs.
\end{abstract}

Keywords: ultrasound therapy, heat therapy, hand, Rheumatoid arthritis.

\section{Introduction}

Multiple health issues might cause dysfunction and pain in human upper limbs. The occurrence of problems is triggered after a long-term repetitive activity, traumatic accidents, infection with viruses and bacteria, etc. Because of high unavoidability of these triggers, statistically high number of people has various diseases that cause pain and other problems in upper limbs. In the 1988 USA National Health Interview Survey, 588,000 reported having tendonitis or related syndromes of the hand, and an estimated 2 million had wrist arthritis with $20 \%$ reporting a resultant major change in work activities, jobs, or missed work days [1,2]. Serious negative effects to patient's daily life raises big need of medical methods to treat or decrease illness symptoms.

One of the possible physical therapy modalities is ultrasound therapy. Multiple studies investigated ultrasound effect on human body resulting in its wide use as a component for physical therapy, for example, treating musculoskeletal disorders such as pain, muscle spasm, joint stiffness, and tissue injury (muscle, tendon, and ligament) [3-6]. One of the studies noted a significant improvement of pain relief and functional performance for patients with chronic low back pain and envisioned possible ultrasound implementations in treatment of other body parts [7]. Langer et al. also investigated knee osteoarthritis and studies showed $39 \%$ in pain level reduction [8-10].

Another possible modality is thermal treatment. In 2004 study to evaluate the efficacy of continuous low-level heat wrap therapy for the treatment of various sources of wrist pain including strain and sprain, tendinosis, osteoarthritis, and carpal tunnel syndrome. After investigating 93 subjects with moderate or greater wrist pain, researchers proved that heat wrap therapy has positive health effects. The results showed that subjects had increased grip strength and relief in pain after therapy [1].

Both modalities were acknowledged providing health benefits, but there is lack of research material providing information on how combination of ultrasound and heat effects human body. To investigate these effects study was conducted. As it was previously mentioned, because of a high need for upper limbs treatment, wrist and hand were taken as a research investigation objects. 


\section{Materials and methods}

The study was performed with a group of 24 patients. The group composition was as listed further: 13 - Rheumatoid arthritis patients, 11 - systemic scleroderma patients mixed with Raynaud's syndrome. The group consisted of 6 males and 18 females. The average age was 57.2 $( \pm 17.9)$ years. Bioethics permission was received for this research from LSMU Bioethics center.

Hand temperature, wrist flexibility and handgrip strength were measured before therapy. Therapy of combined ultrasound and heating was conducted for 15 minutes. After 7 minutes, only temperature at certain points (Fig. 1) was measured. After 15 minutes of therapy, temperature, handgrip strength and wrist flexibility were measured. Furthermore, 7 minutes after the therapy temperature at the same points were measured again. Patients had 5 minutes of adaptation time to room temperature before the therapy and they were not allowed to rub their hands or cover them during the therapy. The temperature was measured in 15 points of hand that are showed in Fig. 1. After 7 minutes of therapy the temperature of the 15 th point was measured nearby, as that point was covered with the device. Paired samples Student's t-tests were used to analyze the gathered data.

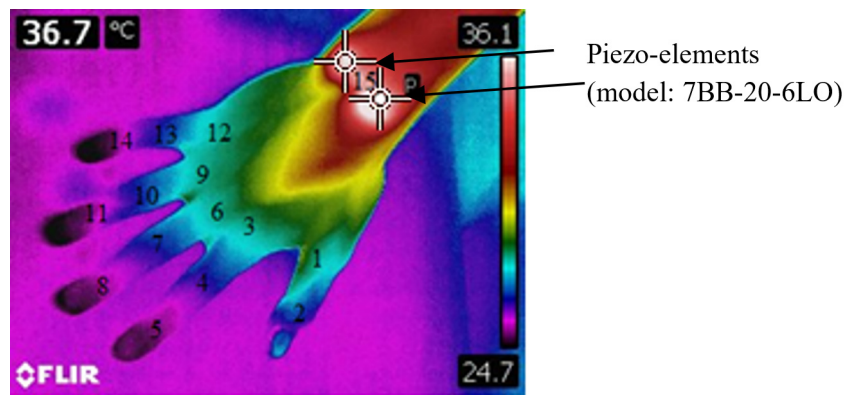

Fig. 1. Hand thermal image with numbered measurement points

The thermographic camera FLIR T450sc was used to capture temperature measurements, ResearchIR software for the analysis of the thermal images, goniometer was used to measure wrist flexibility and dynamometer CAMRY EH101 to test strength. To transfer the high-frequency oscillations to the human body surface, a piezoelectric transducer (model: 7BB-20-6LO) with a resonant frequency of $125 \mathrm{kHz}$, a vibrational amplitude of up to $10 \mu \mathrm{m}$ was used for the hand wrist. Piezo-element emitted highest amount of heat with the selected resonant frequency.

\section{Results}

The gathered data of handgrip strength did not show statistically meaningful improvement after the therapy. However, the rotation movement of all of 4 measured wrist movement axes had improved after the therapy (Fig. 2). As we can see in the figure below, flexibility increased by $8.6 \%$, extension increased by $10.6 \%$, adduction increased by $12.2 \%$, abduction increased by $35.6 \%$.

The comparison of temperature values before the therapy and 15 minutes after that showed that temperature had increased in all measured points. The first seven minutes of therapy had increased temperature in $1 \mathrm{st}, 2 \mathrm{nd}, 3 \mathrm{rd}, 5 \mathrm{th}, 6 \mathrm{th}, 7 \mathrm{th}$, and 15 th measurement points. Last 8 minutes of therapy (from 7 th minute of therapy till 15 th minute of therapy) did not show any significant increase in temperatures, except in 15th point, where the heat therapy was applied. Temperature values before the therapy and 7 minutes after the end of it had showed increase in 3rd, 6th, 9th, 12th, 15th points. Temperature in 7 minutes after the therapy decreased in $2 \mathrm{nd}, 4$ th, 11 th, 13th, 15 th points. Temperature after 7 minutes of the therapy and 7 minutes after the therapy did not show any differences. All averaged values of temperatures at measurement points are showed in Fig. 3. 
All the temperature measurement points had the same pattern - the temperatures were lower in fingers than on hand, without any exceptions. Most of the patients with systemic scleroderma had reported feeling of temperature increase in their fingers and hand. Biggest rise of the temperature was seen in 15 th measurement point $\left(+3.48^{\circ} \mathrm{C}\right)$, where the heat was applied. $3 \mathrm{rd}$, 5 th, 6th measurement points increased temperature by $+1.26^{\circ} \mathrm{C},+1.15^{\circ} \mathrm{C},+1.38{ }^{\circ} \mathrm{C}$ accordingly. All other points increased temperature from $+0.77^{\circ} \mathrm{C}$ to $+1.1^{\circ} \mathrm{C}$.

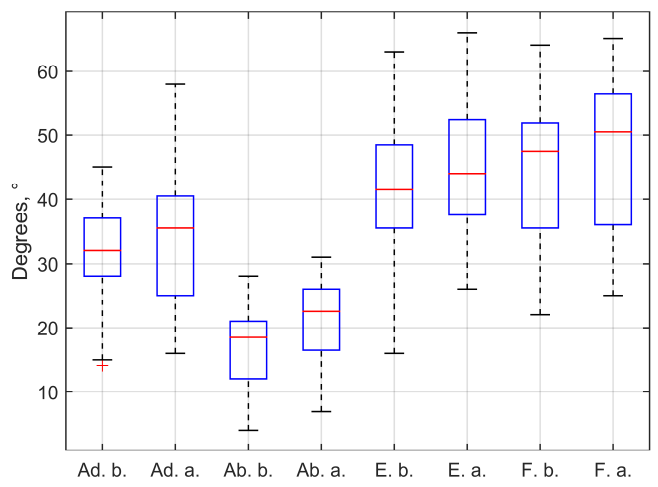

Fig. 2. Comparison of wrist movement before and after the therapy. $A b$-abduction, $A d$ - adduction, $E$ - extension, $F$ - flexion, $a$ - after, $b$ - before

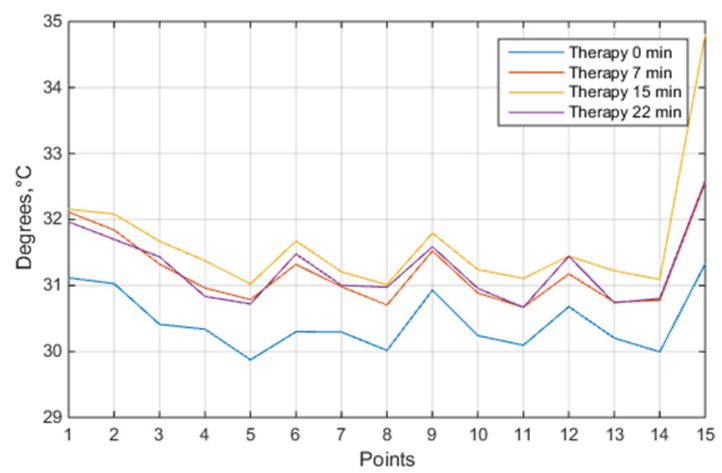

Fig. 3. Temperature values of 15 measurement points in hand: blue line - before the therapy; orange line - during the therapy, after 7 minutes; yellow line - just after the therapy; purple line -7 minutes after the therapy

\section{Discussion and conclusions}

The biggest temperature rise was seen in the first 7 minutes of the therapy. After next 7 minutes of the therapy the temperature rose was not significantly meaningful. This might suggest that heating therapy is most effective in the first 7 minutes. The temperature after 7 minutes of the end of the therapy had dropped only in $2 \mathrm{nd}, 4 \mathrm{th}, 11 \mathrm{th}, 13 \mathrm{th}, 15$ th measurement points. This shows that the therapy we have conducted has lasting and not only momentum effects. This might be analyzed further, how long the risen temperature will last.

The handgrip strength did not show statistically significant improvement. This improvement might not be seen just after a few minutes of therapy. Further researches of the applied therapy for a longer duration should be conducted to analyze improvement in hand grip strength.

The results in Fig. 2 shows that the wrist flexibility had improved in the all measured axis. The biggest improvement can be seen in adduction and abduction. The flexibility values measured after the therapy had reached the averages of healthy people: 20 and 35 degrees respectively. Flexion and extension were improved, but did not reach the averages of healthy people: 70 and 75 
degrees respectively.

In conclusion, it was observed that the ultrasound and heat therapy successfully increased wrist performance. The highest impact of heat to the measured points of hand was seen in the first 7 minutes of therapy. Furthermore, it was observed, that the effect lasts at least 7 minutes after the end of the therapy. This research will be continued and compared to the placebo group of healthy patients.

\section{Acknowledgement}

This research was funded by a Grant (No. SEN-10/15) from the Research Council of Lithuania.

\section{References}

[1] Michlovitz S., Hun L., Erasala G. N., Hengehold D. A., Kurt W. W. Continuous low-level heat wrap therapy is effective for treating wrist pain. Archives of Physical Medicine and Rehabilitation, Vol. 85, 2004, p. 1409-16.

[2] Tanaka S., Wild Dk, Cameron Ll, Fruend E. Association of occupational and non-occupational risk factors with the prevalence of self-reported carpal tunnel syndrome in a national survey of the working population. American Journal of Industrial Medicine, Vol. 32, 1997, p. 550-6.

[3] Rutjes Aws, Nüesch E., Sterchi R., Jüni P. Therapeutic ultrasound for osteoarthritis of the knee or hip. Cochrane Database of Systematic Reviews 2010, https://doi.org/10.1002/14651858.CD003132.pub2.

[4] Knight L. K., Draper O. D. Therapeutic Modalities. The Art and Science, 2th Ed., Philadelphia Lippincott Williams and Wilkins, 2013, p. 252-282.

[5] Wong A. R., Schumann B., Townsend R., Phelps A. C. A Survey of therapeutic ultrasound use by physical therapists who are orthopaedic certifi ed specialists. Physical Therapy, Vol. 87, Issue 8, 2007, p. 986-994.

[6] Moroshita K., Karasuno H., Yokoy Y., Morozumi K., Ogihara H., Ito T., Fijivara T., Fujimoto T., Abe K. Effects of therapeutic ultrasound on intramuscular blood circulation and oxygen dynamics. Journal of the Japanese Physical Therapy Association, Vol. 17, 2014, p. 1-7.

[7] Cantürk F., Dilek Durmua D., Akyol Y., Cengiz K., Terzi T. Effects of therapeutic ultrasound on pain, disability, walking performance, quality of life, and depression in patients with chronic low back pain: a randomized, placebo controlled trial. Archives of Rheumatology, Vol. 25, Issue 2, 2010, p. 82-87.

[8] Langer M. D., et al. Sustained acoustic medicine for the treatment of osteoarthritis of the knee: a randomized, placebo controlled clinical study. Human Research Program Investigator's Workshop, NASA, 2015.

[9] Langer M. D., et al. Novel long duration ultrasound therapy for pain management and biological regeneration. The Military Health System Research Symposium, 2015.

[10] Langer M. D., Levine V., Taggart R., Lewis G. K., Hernandez L., Ortiz R. Pilot clinical studies of long duration, low intensity therapeutic ultrasound for osteoarthritis. Bioengineering Conference, 2014. 